\title{
Efectividad de la atención domiciliaria de enfermería en el seguimiento de prematuros después del alta hospitalaria ${ }^{1}$
}

Institution: Universidad de Costa Rica, Maestría en Enfermería Pediátrica

\section{RESUMEN}

Los prematuros con alta hospitalaria de la Unidad de Cuidado Intensivo Neonatal (UCIN), deben admitirse a un programa de seguimiento para prevenir que tengan complicaciones durante la transición al hogar y se produzcan reingresos hospitalarios. Además, los padres de infantes prematuros experimentan diversos sentimientos y desafíos cuando estos llegan al hogar. El objetivo de este protocolo de práctica clínica basada en la evidencia es analizar la mejor evidencia científica disponible respecto de la efectividad de los programas de atención domiciliaria de enfermería (ADE) para el seguimiento de prematuros egresados de la UCIN. Se utilizó como metodología la práctica clínica basada en la evidencia: inicialmente se planteó una pregunta clínica de tipo pronóstico y se planteó objetivos acordes a esta, a partir de los que se identificó un conjunto de descriptores con los que se realizó la búsqueda de evidencia científica en diferentes bases de datos científicas.

Palabras claves: alta-hospitalaria, enfermería-neonatal, prematuro, seguimiento-domiciliar

\footnotetext{
${ }^{1}$ Fecha de recepción : 5 diciembre del 2017

Fecha de aceptación : 16 de marzo del 2018

${ }^{2}$ Enfermera, estudiante de maestría en Enfermería Pediátrica con énfasis en Neonatología. Universidad de Costa Rica, Costa Rica.

Correo electrónico: anamaromo@gmail.com
} 


\title{
Effectiveness of home nursing care in the follow-up of preterm infants after hospital discharge ${ }^{1}$
}

Institution: University of Costa Rica, Postgraduate Pediatric Nursing

\begin{abstract}
Preterm infants admitted to the Neonatal Intensive Care Unit (NICU) must be admitted to a follow-up program to prevent complications during the transition to the home and hospital readmissions. In addition, parents of premature children experience various feelings and challenges when their child arrives home. The objective of this protocol of clinical practice based on evidence is to analyze the best available scientific evidence regarding the effectiveness of nursing home care programs (ADE) for the follow-up of premature NICU graduates. The clinical practice based on evidence was used as a methodology, initially a clinical question of prognostic type was raised and the objectives according to this were raised, based on which a set of descriptors was identified from which it was made the search for scientific evidence in different scientific databases.
\end{abstract}

Keywords: follow-home, high-hospital, nursing-neonatal, premature

\footnotetext{
${ }^{1}$ Date of receipt : December 5, 2017

Date of acceptance : March 16, 2018

${ }^{2}$ Nurse, master's student in Pediatric Nursing with an emphasis in Neonatology. University of Costa Rica, Costa Rica. E-mail: anamaromo@gmail.com
} 


\title{
Eficácia dos cuidados de enfermagem em casa no seguimento de recém-nascidos prematuros após a alta hospitalar ${ }^{1}$
}

\begin{abstract}
Ana María Rodríguez Molina²
Instituição: Universidade da Costa Rica, Enfermagem pediátrica de pós-graduação

\section{RESUMO}

Os recém-nascidos prematuros admitidos na Unidade de Cuidados Intensivos Neonatais (UCIN) devem ser admitidos no programa de acompanhamento para prevenir os pacientes durante a transição para as readmissões domésticas e hospitalares. Além disso, os pais de crianças prematuras experimentam vários sentimentos e desafios quando seu filho chega em casa. O objetivo deste protocolo é analisar a melhor evidência disponível sobre a eficácia dos programas de cuidados de enfermagem em casa (ADE) para o acompanhamento de graduados prematuros da NICU. A prática clínica baseada na evidência foi utilizada como referência: inicialmente foi levantada uma questão clínica de tipo prognóstico e os objetivos de acordo com isso se basearam em qual conjunto de descritores foi identificado a partir do qual foi feita a busca de evidências científicas em diferentes bases de dados científicas.
\end{abstract}

Palavras-chave: alta hospitalar, enfermagem neonatal, prematuro, siga-domiciliar

\footnotetext{
${ }^{1}$ Data da recepção: 5 de dezembro de 2017

Data de aceitação: 16 de março de 2018

${ }^{2}$ Enfermeira, aluna de mestrado em enfermagem pediátrica com ênfase em neonatologia. Universidade da Costa Rica, Costa Rica.

Correio eletrônico: anamaromo@gmail.com
} 


\section{Revista Electrónica Enfermeria Actual en costa Rica}

\section{INTRODUCCIÓN}

Para la enfermería pediátrica con énfasis en neonatología es relevante garantizar el $4^{\circ}$ derecho de los prematuros que establece que

los recién nacidos de parto prematuro tienen derecho a recibir cuidados de enfermería de alta calidad, orientados a proteger su desarrollo y centrados en la familia. El rol de las enfermeras y los enfermeros tiene un valor primordial en el cuidado de los recién nacidos ${ }^{1}$.

Los prematuros con alta hospitalaria de la Unidad de Cuidado Intensivo Neonatal (UCIN), deben admitirse a un programa de seguimiento para prevenir que tengan complicaciones durante la transición al hogar y se produzcan reingresos hospitalarios, a lo que se suma que los padres de hijos prematuros experimentan diversos sentimientos y desafíos cuando su hijo llega al hogar.

En torno al tema, hacia el 2009, la UNICEF recomendó "las visitas domiciliarias durante la primera semana de vida para aumentar la supervivencia de los recién nacidos"2, mientras que en Checoslovaquia -desde 1949- la visita domiciliaria fue parte del Sistema Distrital de Salud y una práctica común de la enfermera pediátrica en los países europeos ${ }^{3}$.

La preparación para el alta de los prematuros después de la hospitalización en la UCIN, es un proceso que se hace planificadamente y tiene beneficios, por ejemplo, en Europa, específicamente en Inglaterra, los padres mejoran la comprensión del progreso de su bebé y su preparación para el alta, además de que se reduce el número de visitas a los Departamentos de Emergencia de 31 a $20(\mathrm{p}<0.05)$ y los costos de atención médica asociados $(£$ 3400 a $£ 2200 ; \mathrm{p}<0.05)$ después del alta ${ }^{4}$.

De igual forma, en España se ha identificado las experiencias y obstáculos de cuidado que los padres de prematuros tienen cuando participan o no de un programa de alta temprana durante la hospitalización y en el hogar. Para ellos el nacimiento prematuro inicialmente tiene un profundo impacto emocional y consideran que los programas de alta temprana son muy útiles para abordar los aspectos emocionales de la hospitalización y la adquisición de habilidades de atención neonatal ${ }^{5}$.

Paralelamente, cuando el prematuro egresa de la UCIN, la continuidad de su cuidado se hace desde su hogar por medio de la (AD) -como se realiza en España desde el 2002-y se ha observado que los prematuros que tienen este seguimiento han ingresado con rango de peso menor que cuando se inició la $\mathrm{AD}$ y hay menor incidencia de rehospitalizaciones ( $\operatorname{del} 3,4$ al 1,3\%; $\mathrm{p}=0,019)$. Además, se registró un aumento de la lactancia materna exclusiva al alta hospitalaria (del 25,5 al 49,0\%; $p<0,001)^{6}$, además de que se ha demostrado que el prematuro sometido al programa de (ADE) experimenta un aumento de peso superior en el domicilio respecto del hospital y no incrementa su morbilidad ${ }^{6,7}$.

En Estados Unidos, Canadá, Australia y Nueva Zelanda se sintetizó y describió las experiencias de los padres de bebés prematuros después del alta de la UCIN en su transición al hogar, un proceso que requiere apoyo para mejorar la confianza, máxime que la crianza de los hijos prematuros es desafiante, físicamente exigente, altera el rol de los padres, puede ser socialmente perturbadora y se complica aun más por la crianza 


\section{Revista Electrónica Enfermeria Actual en costa Rica}

sobreprotectora $^{8,9}$. Por lo tanto, las enfermeras de UCIN deben esforzarse por proporcionar un apoyo adecuado para los padres a fin de aumentar su confianza en el cuidado después del alta.

Por otro lado, en América del Norte, en Estados Unidos se identificó y describió los errores del cuidado en casa (inasistencia a citas médicas, medicación, alimentación y equipo) y de uso de la atención médica en recién nacidos de alto riesgo después del alta de la UCIN, a través de las visitas domiciliarias de enfermería en el hogar. Anticiparse a estos errores y los desafíos que los padres enfrentan en sus hogares ayuda a los cuidadores a identificar y emplear intervenciones efectivas que son esenciales para optimizar la atención de recién nacidos de alto riesgo durante este periodo de transición ${ }^{10}$.

En Estados Unidos, en un estudio se examinó las preocupaciones y mecanismos de afrontamiento de los padres y madres de niños con muy bajo peso al nacer (MBPN; $<1500 \mathrm{~g}$ ) cuando se trasladan a sus hogares desde la UCIN, entre las que se destaca la incertidumbre generalizada, las preocupaciones médicas persistentes y las de ajuste relacionadas con la pareja que difieren según el sexo ${ }^{11}$. También existe la determinación de hacer lo que sea necesario para garantizar los mejores resultados posibles para sus bebés ${ }^{12}$.

Asimismo, se ha mostrado la efectividad de la intervención temprana del desarrollo después del alta hospitalaria para prematuros ( $<37$ semanas) en el desarrollo motriz o cognitivo. Se evidencia que la intervención mejoró los resultados cognitivos en la edad infantil y hubo poca evidencia en la efectividad en los resultados motores a corto, mediano o largo plazo ${ }^{13}$.

En Centroamérica, se describió las condiciones de egreso de los niños prematuros con más de tres semanas en una Unidad de cuidados intensivos neonatales en México. La mayoría de pacientes prematuros egresan de la UCIN con tratamientos múltiples y prolongados y que es necesario reducir su estancia por la sobrecarga que tienen padres y cuidadores ${ }^{14}$.

En Suramérica, en Brasil, el significado que tiene para las madres de bebés prematuros las visitas domiciliarias favorece el vínculo entre las familias y las enfermeras, disminuye las dudas, miedos y ansiedades de la madre. Se proporciona cuidados en el hogar y se fomenta la adherencia a los cuidados y tratamientos ${ }^{15}$.

En Colombia, se describió las experiencias de los padres de niños prematuros frente al alta de la unidad neonatal: experimentan sentimientos ambivalentes como alegría y temor y el alta es un proceso en el que existen barreras y facilitadores. Así pues, Enfermería debe desarrollar estrategias para involucrarlos tempranamente en el cuidado de sus hijos durante la hospitalización ${ }^{16}$.

Igualmente, se ha descrito el efecto de los programas de intervención temprana y su efecto en la disminución del nivel de estrés en padres de prematuros hospitalizados. Los resultados muestran que, aunque las intervenciones mostraron efectos positivos en la disminución del nivel de estrés en los padres, se evidencia que la educación y el apoyo emocional en conjunto logran un mejor efecto ${ }^{17}$.

De igual modo, se ha descrito las vivencias de madres colombianas de niños prematuros previamente hospitalizados, al asumir el cuidado de ellos en casa. La separación de la diada durante la hospitalización impone limitaciones que impiden que la mujer asuma con tranquilidad y alegría el nuevo rol, por lo que es importante 
empoderar al personal de enfermería de las unidades neonatales para implementar estrategias de contacto temprano y continuo madre-hijo ${ }^{18}$.

No obstante, las percepciones del personal de salud neonatal sobre las barreras y facilitadores para las familias con bebés prematuros que se preparan para el alta de la UCIN incluyen desde los obstáculos a los padres para la visita en la UCIN, las barreras de comunicación, las dificultades relacionadas con el establecimiento de la lactancia materna exitosa, los recursos insuficientes de personal de salud y escasos vínculos entre el hospital y la comunidad. Las madres adolescentes, los padres que trabajan y la familia indígena fueron grupos identificados como particularmente difíciles para participar en la preparación del alta ${ }^{19}$.

En cuanto al primer día después del alta, las madres de los bebés prematuros lo interpretan como un evento que les permite recuperar al hijo, quien no era suyo sino del personal del hospital. La experiencia de cuidarlo la primera noche en casa es vivida con zozobra, angustia y total dedicación; las madres dudan de su capacidad para cuidarlo $^{20}$. Más aún desde el contexto cultural, el significado que tiene para las madres dar cuidado directo al recién nacido prematuro en el hogar es cangurear, masajear, proteger del frío, alimentar al niño, mantener la higiene, conservar la protección del medio, dar amor, suministrar oxígeno suplementario al bebé si lo requiere y saber sobre las señales del niño, máxime que el camino hacia la adaptación materna tiene para las madres un objetivo: "criar el niño",

En el caso de Costa Rica, se diseñó un programa de intervención de enfermería neonatal para la integración en el hogar de infantes de alto riesgo atendidos en el Servicio de Neonatología del Hospital Dr. Rafael Ángel Calderón Guardia. Al realizar este proyecto se evidencia que una adecuada preparación a la familia, para la transición del hospital al hogar repercute en la disminución de costos, es segura y favorece una mejor calidad de vida $^{1}$.

Por lo anterior, es evidente que existen trabajos de investigación relacionados con la planificación del alta en UCIN, programas de seguimiento interdisciplinario y la ADE para los prematuros egresados de la UCIN. Sin embargo, la ADE únicamente está establecida como un programa en España y Brasil donde se realizan visitas en el hogar para esta población. Asimismo, varios estudios establecen las experiencias de los padres en ese periodo de transición de la UCIN al hogar y por eso es necesario que reciban apoyo no solo antes, sino después del alta neonatal.

Para abordar el tema del seguimiento de los prematuros egresados de la UCIN por medio de los programas de Atención Domiciliaria de Enfermería (ADE), es importante referirse a los Objetivos del Desarrollo del Milenio para el 2015 en su cuarto objetivo se plantea "reducir la mortalidad de los niños menores de 5 años" 22 ya que, aunque ha disminuido la tasa reportada, los recién nacidos son quienes aún presentan la más alta causada por la prematurez. Entonces, los recién nacidos prematuros que se hospitalizan en las (UCIN) y sus familias son quienes requieren una preparación antes del alta y una continuidad para darle seguimiento durante ese proceso de transición de la UCIN al hogar, para evitar complicaciones y reingresos. Además, para la madre y el padre no es suficiente únicamente el entrenamiento y la información que reciben en la UCIN previos al egreso.

\footnotetext{
${ }^{1}$ Angulo B, Fernández N, Molina CL. Intervención de Enfermería Neonatal en la Integración de niños y niñas de Alto riesgo atendidos en el servicio de neonatología del Hospital Dr. Rafael Ángel Calderón Guardia [Trabajo Final de Maestría]. Universidad de Costa Rica; 2006. Disponible en http://repositorio.sibdi.ucr.ac.cr:8080/jspui/handle/123456789/1117
} 
Por otro lado, también se han reseñado experiencias y obstáculos que tienen los padres y las madres, que han sido percibidos por el personal de salud como facilitadores y barreras para la preparación del egreso del prematuro de la UCIN ${ }^{5,19}$. En consecuencia, es un momento que genera miedo, angustia y otros sentimientos que se pueden atenuar con los programas de ADE en el Hogar desde el primer día que egresan.

Es cierto, que existen programas de seguimiento para el prematuro ambulatoriamente como el Método Canguro $^{23}$, pero se realiza desde el hospital y no desde el hogar, donde se puede tener una percepción real del contexto donde va a llevar el niño después de su salida de la UCIN. También se ha llegado a conocer que el seguimiento de los prematuros después del alta neonatal difiere entre los países.

Por otro lado, la revisión de la literatura evidencia un abordaje extenso del tema y se puede leer ampliamente las experiencias, vivencias y significados que representan para las madres, y en algunos estudios para los padres, la transición del prematuro desde la UCIN al hogar y los errores que pueden producirse en el hogar ${ }^{10,14}$. Sin embargo, son pocos los estudios que muestran la efectividad de los programas de seguimiento a nivel domiciliario realizados por enfermería, dado que la mayor parte de las intervenciones están orientadas a la preparación para el egreso del prematuro de la UCIN.

En España está establecida la Atención Domiciliaria en el Hogar y en Brasil como visitas domiciliarias, motivo por el que se ha desarrollado estudios para evaluar su evolución, efectividad y significado para las madres $6,7,15$, además de que se ha evaluado la efectividad de las intervenciones tempranas en los prematuros y su influencia en el desarrollo cognitivo ${ }^{13}$. Lo anterior es relevante en cuanto a que, una vez que llega el momento del alta de la UCIN, se generan preocupaciones y mecanismos de afrontamiento por los padres ${ }^{11}$; inclusive, en la literatura se reseñan experiencias, vivencias en la preparación para el cuidado al infante prematuro posterior al egreso hospitalario y los significados de las madres o padres en el hogar al cuidarlos $8,9,12,16,18,20,21$.

Por eso, es importante realizar el cuidado de enfermería para el prematuro desde su hogar apoyando a su familia, con el fin de darle continuidad al seguimiento que se le debe realizar para evitar complicaciones y que reingrese de nuevo al hospital. Cuando el prematuro está en condiciones de dejar la UCIN e irse con sus padres, ellos tienen que asumir en su totalidad su rol y recuperar a su bebé después de haberse separado de este por su inmadurez y los riesgos que le implicó un nacimiento antes de tiempo. Tal momento debería iniciar desde la UCIN, siguiendo una transición planificada, debido a que los padres deben tener una preparación antes del egreso para facilitarles ese proceso, en vez de encontrarse con barreras y obstáculos, máxime que -al llegar al hogar- el estrés, angustia, temor, incertidumbre, inseguridad que tienen los padres ${ }^{12,17,21}$, se puede disminuir al tener el respaldo de un profesional de enfermería que les acompañe y les ayude a resolver dudas que de pronto no surgieron antes de irse de la UCIN y que emergen posteriormente.

Por otra parte, con la intervención de enfermería desde el hogar, partiendo de un programa de seguimiento, se disminuye los costos de las instituciones de salud, porque la estancia hospitalaria se puede reducir sin riesgo, al tener la certeza que hay un profesional de enfermería dándole educación y apoyo a la familia del prematuro en el hogar. También hay menos consultas por emergencias y a su vez reingresos que se pudieron evitar al conocer el contexto donde vive el prematuro en su hogar y la adherencia que han tenido los padres a las medidas de cuidado y al tratamiento, sin cometer errores ${ }^{10}$. De la misma manera, se detectarían a tiempo dificultades en la lactancia materna, porque con los programas de ADE los prematuros aumentan de peso ${ }^{6}$. 
Igualmente, los padres aumentan su confianza porque el cuidado que reciben en su hogar es personalizado para su hijo, se estrecha el vínculo entre padres y enfermero o enfermera. El cuidado del prematuro representa un gran desafío para los padres, porque es exigente, se altera la dinámica de la pareja y de la familia, llegando a veces a una crianza sobreprotectora ${ }^{8}$. No es exclusivamente de la madre el cuidado del prematuro, sino de la familia y sigue siendo responsabilidad de las instituciones de salud. El profesional de enfermería es quien da educación a la madre para que ella y la familia sepan cómo se debe cuidar al prematuro en casa, máxime para aquellos padres en situaciones especiales como adolescentes, o para quienes por razones laborales no pudieron estar en el hospital recibiendo la preparación para el alta, que viven en áreas rurales o son parte de comunidades indígenas y el desplazamiento se les dificulta ${ }^{19}$.

La evidencia señala que en países como Brasil y España los programas de seguimiento para prematuros que realizan profesionales de enfermería en el hogar han sido muy exitosos. Sin embargo, en otros contextos la continuidad de los programas después del alta, solo se lleva a cabo en el hospital por otros profesionales de salud (eonatólogo, pediatra, neumólogo, terapeuta ocupacional, fonoaudiólogo, entre otros), mientras que los padres tienen que llevar con alta frecuencia a los prematuros a las citas programadas al egreso de la UCIN. Sin embargo, con la ADE, el profesional de enfermería especialista en neonatología estaría en capacidad de realizar la valoración y seguimiento del recién nacido, desde un ámbito diferente -el hogar- donde se puede obtener un conocimiento real de las necesidades y condiciones con la familia.

Al respecto, en Costa Rica, en el Hospital Nacional de Niños Dr. Carlos Sáenz Herrera, existen programas de seguimiento al hogar en los que la enfermería tiene un rol importante en la consulta propia y ejecuta visitas semanales en el hogar, así como en las escuelas en donde sensibiliza a los docentes y estudiantes y hace referencia a un nivel de mayor complejidad, de ser pertinente, según el caso de cada niño. Estos programas se hacen desde que son recién nacidos hasta que llegan a la adolescencia, únicamente si presentan las siguientes situaciones: falla para progresar, parálisis cerebral infantil (PCI), cardiopatías, disfunción motora-oral, incoordinación cricofaríngea, heparinización y hemodiálisis. Sin embargo, no se ha establecido un programa exclusivamente dirigido a los recién nacidos prematuros, sino que ellos son valorados en el neurodesarrollo en la Unidad de Desarrollo hospitalaria por un especialista.

Finalmente, enfermería finiquita su relación de cuidado con el prematuro apenas egresa de la UCIN. No obstante, podría retomar su papel para desarrollar programas de seguimiento en el domicilio, durante la transición de la UCIN al hogar, fortalecer con su familia el cuidado del prematuro, prevenir complicaciones y empoderar a los padres y/o demás familiares.

Para esta revisión breve, es importante documentar con la mejor evidencia disponible, las experiencias que se suscitan en otros contextos respecto de los programas de visita domiciliar, que sirvan de referente para llevar a cabo la investigación y las reestructuraciones fundamentadas científicamente en los programas establecidos en Costa Rica o crear nuevos programas que fortalezcan la educación de los padres de los niños prematuros, disminuyan sus temores y que el cuidado que se da sea exitoso para su ulterior desarrollo. Por lo tanto, es necesario obtener la mejor evidencia sobre la efectividad de los programas de seguimiento domiciliario de Enfermería en el hogar para los prematuros que egresan de UCIN. 
Por lo anterior, se ha propuesto el objetivo de analizar la mejor evidencia científica disponible respecto de la efectividad de los programas de atención domiciliaria de enfermería para el seguimiento de prematuros egresados de la Unidad de Cuidado Intensivo Neonatal (UCIN).

\section{MÉTODO}

Se aplicará la metodología de práctica clínica basada en la evidencia, la cual sigue cinco pasos: 1 . Establecimiento de una pregunta clínica 2. Búsqueda de la mejor evidencia científica 3. Análisis crítico de la mejor evidencia encontrada 4. Transferencia de la evidencia científica y 5. Evaluación de la transferencia de la evidencia científica.

\section{Pregunta clínica}

La pregunta de revisión es la siguiente: ¿Cuál es la efectividad de los programas de atención domiciliaria de enfermería para el seguimiento de los prematuros egresados de la Unidad de Cuidado Intensivo Neonatal (UCIN)? Lo anterior se desglosa en el siguiente acrónimo PCC:

\begin{tabular}{|l|l|l|}
\hline \multicolumn{1}{|c|}{ Población } & \multicolumn{1}{c|}{ Concepto } & \multicolumn{1}{c|}{ Contexto } \\
\hline $\begin{array}{l}\text { Prematuros con alta } \\
\text { hospitalaria }\end{array}$ & $\begin{array}{l}\text { Atención domiciliaria de } \\
\text { enfermería }\end{array}$ & $\begin{array}{l}\text { Unidad de cuidado } \\
\text { intensivo neonatal }\end{array}$ \\
\hline
\end{tabular}

El tipo de pregunta se clasifica como una pregunta de "alcance", la cual se elabora cuando se quiere determinar el alcance de un cuerpo de literatura sobre un tema, por lo que se mapea los conceptos clave sobre un área de investigación.

\section{Criterios de inclusión}

Los criterios de inclusión para recuperar la evidencia son los siguientes:

Población: se considerará estudios que incluyan recién nacidos prematuros con alta hospitalaria independientemente de los días de nacidos, sexo o edad gestacional.

Concepto: se incluirá estudios que hagan referencia a la atención domiciliaria de enfermería para los recién nacidos con condición de prematuridad, así como los que se relacionen con programas o intervenciones de enfermería realizadas como atención domiciliaria o visita domiciliaria. Se excluye los elaborados en la Unidad de Cuidado Intensivo o por otros profesionales de salud

Contexto: se incluirá estudios que aborden el tema de los recién nacidos pretérmino que hayan estado hospitalizados en una unidad de cuidado intensivo neonatal. 


\section{Revista Electrónica Enfermeria Actual en costa Rica}

\section{Tipos de estudios}

Se considerará los estudios publicados tanto experimentales como no experimentales; los diseños incluyen ensayos clínicos, ensayos clínicos aleatorizados, series de tiempo, estudios observacionales con cohortes prospectivos y retrospectivos, casos y controles y estudios descriptivos que se complementan con reportes de casos individuales, estudios con diseño cualitativo, cuya publicación esté en idioma español, inglés y portugués.

\section{Estrategia de búsqueda}

La estrategia de búsqueda tendrá como objetivo encontrar estudios publicados, la cual seguirá tres pasos: inicialmente se llevará a cabo una búsqueda limitada en MedLine, Google Scholar, Science direct, EBSCO seguida de un análisis de las palabras textuales contenidas en el título y el resumen, y de los términos de índice utilizados para describir cada artículo. Luego se realizará una segunda búsqueda utilizando para ello los descriptores planteados en esta revisión en las bases de datos Clinical key, EMBASE, Lilacs, Medline vía EBSCO, Ovid, Pubmed, Scielo, Science Direct y Scopus. En tercer lugar, se buscará estudios adicionales en la lista de referencias de todos los informes y artículos identificados. Se incluirá toda la información disponible entre el 2012 y el 2017.

\section{Descriptores de búsqueda}

Para la búsqueda de información se utilizarán los descriptores, en idioma español, inglés y portugués expuestos en la tabla1, utilizando AND, OR, NOT.

Tabla 1. Descriptores para la búsqueda de información

\begin{tabular}{l|l|l}
\hline \multicolumn{1}{c}{ Español } & \multicolumn{1}{c}{ Descriptores } \\
\hline \multicolumn{1}{c|}{ Inglés } & \multicolumn{1}{c}{ Portugués } \\
\hline Prematuro con alta hospitalaria & pediatric home care for nursing & prematuro com alta hospitalar \\
\hline Atención del prematuro en el hogar & care for the premature at home & atenção à criança prematura no domicílio \\
\hline Seguimiento al hogar & home monitoring & acompanhamento na casa \\
\hline Visita domiciliaria pediátrica de enfermería & nursing home pediatric visit & visita domiciliária pediátrica para enfermagem \\
\hline $\begin{array}{l}\text { Atención domiciliaria pediátrica por } \\
\text { enfermería }\end{array}$ & pediatric home care for nursing & $\begin{array}{l}\text { Cuidados domiciliários pediátricos para } \\
\text { enfermagem }\end{array}$ \\
\hline Visita al prematuro por enfermería & visit to the premature by nursing & visita ao filho prematuro por enfermagem \\
\hline $\begin{array}{l}\text { Programa de atención domiciliaria al } \\
\text { prematuro }\end{array}$ & $\begin{array}{l}\text { Home care program for premature } \\
\text { babies }\end{array}$ & $\begin{array}{l}\text { programa de cuidados domiciliários para o } \\
\text { prematuro }\end{array}$ \\
\hline
\end{tabular}

Fuente: elaboración propia

\section{Selección de los estudios}

Luego de la búsqueda de información, todas las citas identificadas se recopilará y se cargará en el software bibliográfico Mendeley y se eliminará los duplicados. Se examinará los títulos y los resúmenes para evaluarlos según los criterios de inclusión para la revisión. Los estudios que cumplan con los criterios de inclusión se recuperará en su totalidad y se importará sus detalles en una matriz de datos de Excel. El texto completo de los 


\section{Revista Electrónica Enfermeria Actual en costa Rica}

estudios seleccionados se recuperará y evaluará en detalle en relación con los criterios de inclusión. Se excluirá los estudios de texto completo que no cumplan con los criterios de inclusión y se proporcionará los motivos de la exclusión en un apéndice del informe de la revisión final.

Los resultados de la búsqueda se anotará en su totalidad en el informe final y se presentará en un diagrama de flujo PRISMA 2009 en su versión en español.

\section{Extracción de los datos}

Los datos se extraerá de los artículos incluídos en la revisión de forma manual. Los datos extraídos incluirá detalles específicos, como por ejemplo: año, autor, título, tipo y métodos del estudio, participantes, tipo de intervención, resultado principal y secundario de importancia para la pregunta clínica planteada y los objetivos específicos.

Se contactará a los autores de los documentos para solicitar datos faltantes o adicionales cuando sea necesario.

\section{Evaluación de la calidad metodológica}

El análisis crítico de la evidencia se elaboró a partir de las etapas que mencionan para su desarrollo Rojas, Cubero y Leiva ${ }^{24}$, a partir de la lectura crítica, la cual es "discriminar aquella que carece de validez debido a: deficiente rigurosidad metodológica, sesgos, claridad en los procedimientos, inadecuado diseño y por una inadecuada respuesta a los objetivos planteados en un inicio dentro de la investigación" ${ }^{24}$.

La calidad de la información y su pertinencia para dar respuesta a la pregunta planteada en la investigación se hará a través de la plataforma de fichas de lectura crítica 2.0 (FLC 2.0) que permite analizar la calidad o validez de los artículos científicos y que se define como "una aplicación web diseñada para apoyar el desarrollo de revisiones sistemáticas de la evidencia científica proporcionando un apoyo a la evaluación de la calidad de los estudios científicos y a la síntesis de la evidencia,"25.

\section{Síntesis de datos}

Los resultados de esta revisión breve serán sintetizados y presentados en forma de tabla de evidencia y resumen narrativo de acuerdo con los objetivos específicos para su análisis posterior.

\section{Conflicto de intereses}

La autora declara que no tiene conflictos de tipo personal, comercial, académico, político o financiero. 


\section{Agradecimientos}

A la Doctora Ligia Patricia Rojas Valenciano por su asesoría y apoyo para el desarrollo de la investigación.

\section{REFERENCIAS BIBLIOGRÁFICAS}

1. Ravalli MJ, Ortiz Z. Decálogo del prematuro [Internet]. Buenos Aires, Argentina: UNICEF- Argentina; 2011. 9 p. Disponible en: https:/www.unicef.org/argentina/spanish/Decalogo2011.pdf

2. OMS, UNICEF. Visitas domiciliarias al recién nacido: una estrategia para aumentar la supervivenciaDeclaración conjunta OMS/UNICEF [Internet]. OMS; 2009. 8 p. Disponible en: http://apps.who.int/iris/bitstream/10665/70057/1/WHO_FCH_CAH_09.02_spa.pdf

3. Shönbauerová A, Boledovicova M. The role of paediatric nurse in home visiting of newborns and infants in the past - literary survey. Kontakt. 2015;17(4):e211-8.

4. Ingram JC, Powell JE, Blair PS, Pontin D, Redshaw M, Manns S, et al. Does family-centred neonatal discharge planning reduce healthcare usage? A before and after study in South West England. BMJ Open. 2016;6(3):e010752.

5. Toral-López I, Fernández-Alcántara M, González-Carrión P, Cruz-Quintana F, Rivas-Campos A, PérezMarfil N. Needs Perceived by Parents of Preterm Infants: Integrating Care Into the Early Discharge Process. J Pediatr Nurs 2016;31(2):e99-108.

6. Figueras J, Esqué MT, Arroyo L, Bella J, Álvarez R, Carbonell X. Evolución de la atención a domicilio para niños prematuros y de bajo peso de nacimiento desde 2002 hasta 2014. An Pediatr. 2016;85(3):134-41. http://dx.doi.org/10.1016/j.anpedi.2016.01.022

7. Álvarez R, Lluch MT, Figueras J, Esqué MT, Arroyo L, Bella J, et al. Evolución del peso del prematuro con alta precoz y atención domiciliaria de enfermería. An Pediatría. 2014;81(6):352-9.

8. Adama EA, Bayes S, Sundin D. Parents' experiences of caring for preterm infants after discharge from Neonatal Intensive Care Unit: A meta-synthesis of the literature. J Neonatal Nurs. 2016;22(1):27-51.

9. Boykova M. Life After Discharge: What Parents of Preterm Infants Say About Their Transition to Home. Newborn Infant Nurs Rev. 2016;16(2):58-65.

10. Patel R, Nudelman M, Olarewaju A, Pooley SW, Jegatheesan P, Song D, et al. Homecare and Healthcare Utilization Errors Post-Neonatal Intensive Care Unit Discharge. Adv Neonatal Care. 2017;17(4):258-64.

11. Garfield CF, Lee Y, Kim HN. Paternal and Maternal Concerns for Their Very Low-Birth-Weight Infants Transitioning From the NICU to Home. J Perinat Neonatal Nurs. 2014;28(4). 
12. Phillips-Pula L, Pickler R, McGrath JM, Brown LF, Dusing SC. Caring for a Preterm Infant at Home. J Perinat Neonatal Nurs. 2013;27(4):335-44.

13. Spittle AJ, Orton J, Doyle LW, Boyd R. Early developmental intervention programs post hospital discharge to prevent motor and cognitive impairments in preterm infants. Cochrane Database Syst Rev. 2007;206(2):145-206.

14. Agami-Micha S, Iglesias-Leboreiro J, Bernárdez-Zapata I, Rendón-Macías ME. Condiciones al egreso de niños prematuros atendidos en una Unidad de Cuidados Intensivos Neonatales. Rev Mex Pediatría. 2012;79:716.

15. Santos LC, Balaminut T, Souza SNDH, Rossetto EG. Perception of premature infants' mothers on home visits before and after hospital discharge. Investig y Educ en Enfermería. 2014;32(3):393-400.

16. Osorio S, Ochoa S, Semenic S. Preparing for post-discharge care of premature infants: Experiences of parents. Investig y Educ en Enfermería. 2017;35(1).

17. Villamizar B, Vargas C, Gómez OR. Metaanálisis: efecto de las intervenciones para disminuir el nivel de estrés en padres de prematuros. Aquichan. 2016;16(3):276-95.

18. Ocampo M, Castillo E. Caring for a premature child at home: from fear and doubt to trust. Text Context Nurs. 2014;23(4):828-35. http://dx.doi.org/10.1590/0104-07072014003280013

19. Raffray M. Barriers and facilitators to preparing families with premature infants for discharge home from the neonatal unit . Perceptions of health care providers. 2014;32(3):379-92.

20. Castillo E, Ocampo MP. Retrieving the offspring and caring for it during the first night at home. Investig Educ en Enferm. 2013;31(3):354-63.

21. Castiblanco N, Muñoz L. Visión de las madres en el cuidado del hijo prematuro en el hogar. Av en enfermería. 2011;(1):120-9.

22. ONU. Podemos Erradicar la pobreza: Objetivos del Desarrollo del Milenio y más allá del 2015 [Internet]. Objetivo 4: Reducir la mortalidad en los niños menores de 5 años. Disponible en:

http://www.un.org/es/millenniumgoals/childhealth.shtml

23. Charpak N, Ruíz JG, Angel MaI, Duque JS, García C. Lineamientos técnicos para la implementación de Programas Madre Canguro en Colombia. Colombia; 2012 p. 122.

24. Rojas-Valenciano L, Cubero- Alpízar C, Leiva- Dïaz V. Investigación secundaria: utilización de la mejor evidencia en la práctica clínica. 3ra ed. CIEBE, UCR, editores. Costa Rica; 2017. 175. 
25. López de Argumedo M, Reviriego E, Andrío E, Rico R, Sobradillo N, Hurtado I. Revisión externa y validación de instrumentos metodológicos para la Lectura Crítica y la síntesis de la evidencia científica. Madrid: Servicio de Evaluación de Tecnologías Sanitarias del País Vasco (Osteba); 2006. Disponible en http://www.lecturacritica.com/es/acerca.php 